\title{
Uma Proposta de Hibridização do Método Simulated Annealing na Minimização de
} Funções Não Lineares

\section{A Proposed Hybridization of the Simulated Annealing Method in the Minimization of Non- linear Functions}

Mateus Braga Oliveira ${ }^{1}$, Joviana Sartori de Souza ${ }^{2}$, Thiago Jordem Pereira ${ }^{3}$

\section{RESUMO}

Este trabalho tem como principal objetivo a determinação de minimizadores globais de funções não lineares por meio do método Simulated Annealing. Serão utilizadas funções não lineares com 20 e 30 variáveis, para verificação da eficiência dos métodos apresentados na minimização de funções não lineares de várias variáveis. Também será proposto uma hibridização, com a inserção do método determinístico Hooke Jeeves ao longo das iterações do método Simulated Annealing. Assim, é feito a comparação de todos os métodos testados para as funções não lineares, verificando o tempo gasto na obtenção dos minimizadores globais das funções e a eficiência dos métodos. A inserção do Hooke Jeeves é feita para verificar a influência da realização de uma diferente busca nas soluções geradas pelo Simulated Annealing. Deste modo, o foco deste trabalho é mostrar uma metaheurística de otimização e um método híbrido para a determinação dos minimizadores globais para funções não lineares de várias variáveis. Com os resultados apresentados, será realizada uma comparação, verificando qual metodologia foi mais robusta na determinação dos mínimos globais para os problemas testados. Assim, pode-se analisar a influência do método Hooke Jeeves, de acordo com sua inserção ao longo das iterações, no desempenho do método Simulated Annealing.

Palavras-chave: Simulated Annealing. Hibridização. Funções não lineares. Hooke Jeeves.

\section{ABSTRACT}

This work has as main objective the determination of global minimizers of nonlinear functions through the Simulated Annealing method. Nonlinear functions with 20 and 30 variables will be used to verify the efficiency of the methods presented in the minimization of nonlinear functions of several variables. Also a hybridization will be proposed, with the insertion of the deterministic method Hooke Jeeves along the iterations of the Simulated Annealing method. Thus, a comparison of all the methods tested for nonlinear functions is performed, verifying the time taken to obtain the global function minimizers and the efficiency of the methods. The insertion of Hooke Jeeves is done to verify the influence of the realization of a different search in the solutions generated by Simulated Annealing. Thus, the focus of this work is to show an optimization metaheuristic and a hybrid method for the determination of global minimizers for nonlinear functions of several variables. With the results presented, a comparison will be made, verifying which methodology was more robust in determining the global minimums for the problems tested. Thus, it is possible to analyze the influence of the Hooke Jeeves method, according to its insertion along the iterations, in the performance of the Simulated Annealing method.

Keywords: Simulated annealing, Hybridization, Nonlinear Functions, Hooke Jeeves. 


\section{INTRODUÇÄAO}

Neste trabalho, uma comparação entre algoritmos de otimização na busca de minimizadores globais de funções de várias variáveis é realizada, com o objetivo de verificar qual dos métodos abordados é mais eficiente na resolução do problema proposto. A metaheurística estocástica Simulated Annealing é utilizada na minimização de funções não lineares de 20 e 30 variáveis. Além disso, é proposto uma hibridização, onde serão utilizados o método Simulated Annealing e o método determinístico Hooke Jeeves. É feita a inserção do método Hooke Jeeves ao longo das iterações do método Simulated Annealing.

Deste modo, o foco deste trabalho é apresentar um método estocástico (Simulated Annealing), propor sua hibridização com um método determinístico (Hooke Jeeves), e comparar os resultados obtidos para funções de várias variáveis. A partir desses resultados podemos analisar a eficiência dos métodos abordados e a contribuição da hibridização realizada.

\subsection{Funções Não Lineares}

A partir de agora, são apresentadas as funções testadas com a metodologia proposta. Essas funções não lineares estão presentes em BACK (1996) e MOLGA (2005). Foram realizados testes com funções de 20 e de 30 variáveis. Para ilustrar a dificuldade de minimização dessas funções, são apresentados os gráficos tridimensionais para cada um dos problemas.

\section{Função Ackley}

A função Ackley é amplamente utilizada para testes de algoritmos de otimização. $\mathrm{Na}$ sua forma bidimensional, caracteriza-se por uma região externa quase plana, e um grande orifício no centro. A função representa um risco para algoritmos de otimização, particularmente algoritmos de subida, por ficar preso em um de seus muitos mínimos locais.

A função geralmente é avaliada no hipercubo $x_{i} \in[-32,78632,786]$, para todo i $1, \ldots, d$, embora possa também ser restrito a um domínio menor.

$$
f(x)=-a \cdot \exp \left(-b \sqrt{\frac{1}{d} \sum_{1}^{d} x_{i}^{2}}\right)-\exp \left(-b \sqrt{\frac{1}{d} \sum_{1}^{d} \cos \left(c x_{i}\right)}\right)+a+\exp (1)
$$

onde $\mathrm{a}=20, \mathrm{~b}=0,2, c=2 \pi$ e d é a Dimensão da função.

O Mínimo global da função Ackley é: $f\left(x^{*}\right)=0 \mathrm{em} x=(0, \ldots, 0)$. 


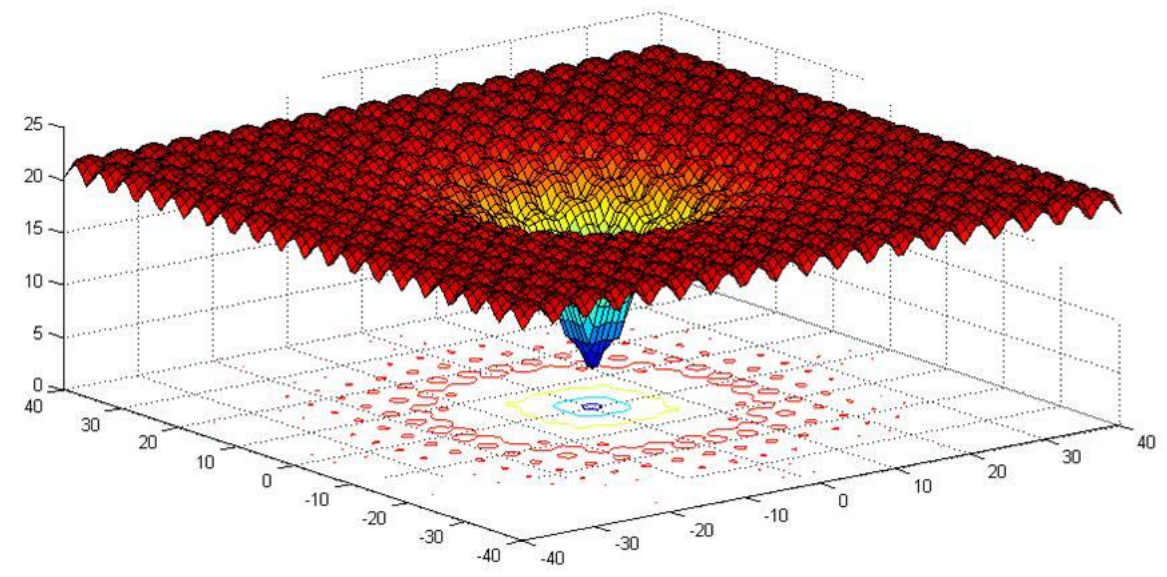

Figura 1. Função Ackley e seu mínimo global.

\section{Função Levy}

Nesta função foi considerada 20 e 30 dimensões. Ela é normalmente calculada na região $x_{i} \in[-10,10]$ para $\mathrm{i}=1, \ldots, \mathrm{d}$.

$$
f(x)=\operatorname{sen}^{2}\left(\pi \omega_{i}\right)+\sum_{i=1}^{d-1}\left(\omega_{i}-1\right)^{2}\left[1+10 \operatorname{sen}^{2}\left(\pi \omega_{i}+1\right)\right]+\left(\omega_{d}-1\right)^{2}\left[1+\operatorname{sen}^{2}\left(2 \pi \omega_{d}\right)\right],
$$

onde $\omega_{i}=1+\frac{x_{i}-1}{4}$.

O Mínimo global da função Levy é dado por: $f\left(x^{*}\right)=0$ em $x=(1, \ldots, 1)$.

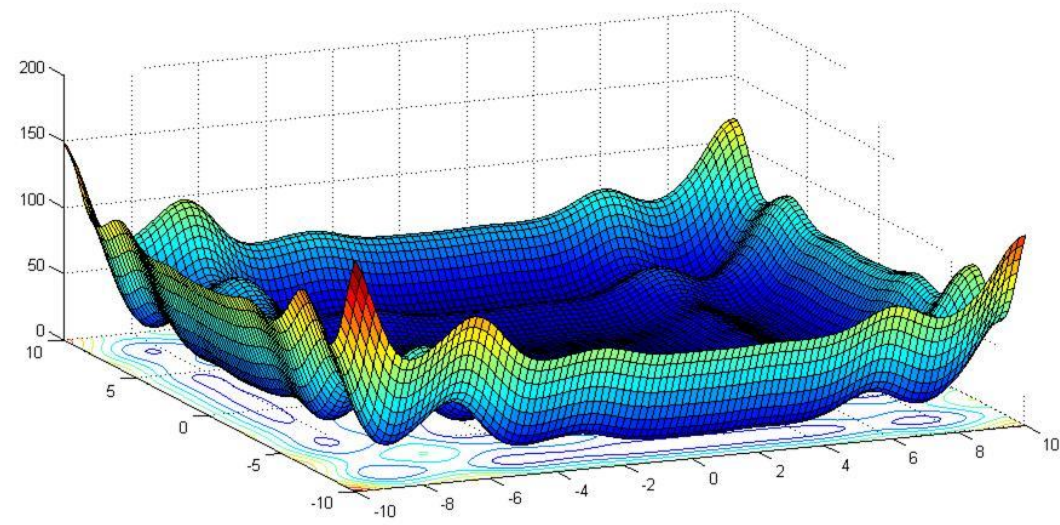

Figura 2. Função Levy e seu ponto de mínimo global.

\section{Função Schwefel}

É uma função complexa com muitos mínimos locais. Esta função é calculada na região $x_{i} \in[-500,500]$ para $\mathrm{i}=1, \ldots, \mathrm{d}$. Com d dimensões. 


$$
f(x)=418,9829 d-\sum_{i=1}^{d} x_{i} \operatorname{sen}\left(\sqrt{\left|x_{i}\right|}\right)
$$

O Mínimo global da função Schwefel é: $f\left(x^{*}\right)=0$ em $x=(420.9687, \ldots, 420.9687)$.

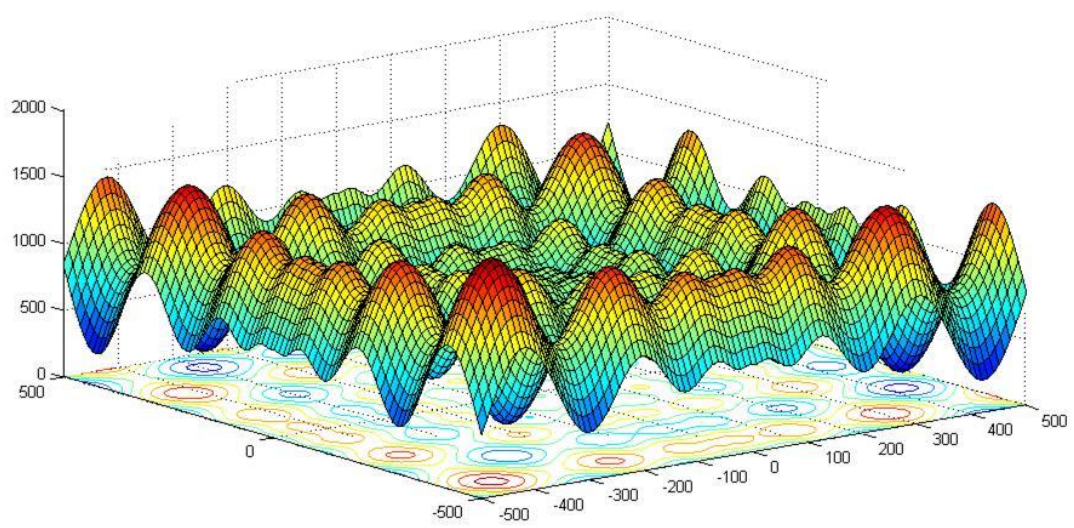

Figura 3. Função Schwefel, d=2.

\section{Função Styblinski-Tang}

Esta função possui $d$ dimensões. Neste trabalho, foram considerados $d=20$ e $d=30$. A função usualmente é calculada no hipercubo $x_{i} \in[-5,5]$ para $\mathrm{i}=1, \ldots, \mathrm{d}$.

$$
f(x)=\frac{1}{2} \sum_{i=1}^{d}\left(x_{i}^{4}-16 x_{i}^{2}+5 x_{i}\right)
$$

O Mínimo global é dado por: $f\left(x^{*}\right)=-39,16599 d$ em $x=(-2.903534, \ldots,-2.903534)$.

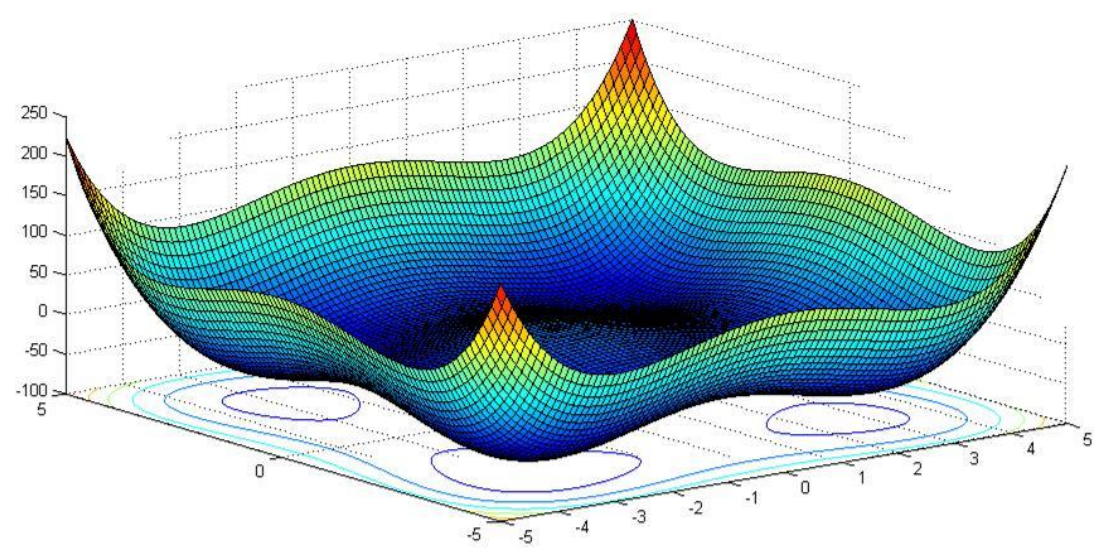

Figura 4. Função Styblinski-Tang. 


\section{MATERIAIS E METODOS}

O objetivo deste trabalho é a minimização das funções não lineares já apresentadas. Estas funções serão minimizadas por meio do método Simulated Annealing. Também, propõe-se neste trabalho a hibridização do método Simulated Annealing com o método determinístico Hooke Jeeves. Visa-se verificar a influência que pode ter o método Hooke Jeeves, um método de características distintas, em conjunto com o método Simulated Annealing. Essa influência será analisada por meio de tempo computacional e número de iterações para obtenção dos minimizadores globais de funções não lineares. A partir de agora, descrevem-se os métodos trabalhados.

\subsection{O Método Simulated Annealing}

O Método Simulated Annealing (Recozimento Simulado) (SA), foi proposto por KIRKPATRICK et al. (1983). Em COSTA (2012) descreve-se o método Simulated Annealing, onde este método simula o recozimento de metais. O princípio do método sugere que, com uma redução rápida de temperatura, gera-se produtos metaestáveis, e com uma redução lenta de temperatura, os produtos são mais estáveis e fortes estruturalmente. Há também uma variação na energia interna dos produtos que são gerados.

Assim, o Simulated Annealing é um método que facilita a obtenção de minimizadores globais de funções não lineares. Ao longo de suas iterações, quando se tem uma temperatura alta considerada no método, é possível aceitar soluções onde ocorre a piora dos valores da função objetivo. Esta aceitação permite que o método fuja de minimizadores locais. Com o decorrer das iterações, onde a temperatura é reduzida, a chance de aceitação de uma solução de piora é reduzida, o que faz com que o método localize uma solução e se aproxime dela. As soluções são atualizadas por meio das características aleatórias do método.

O algoritmo a seguir ilustra a fundamentação do Método Simulated Annealing: 


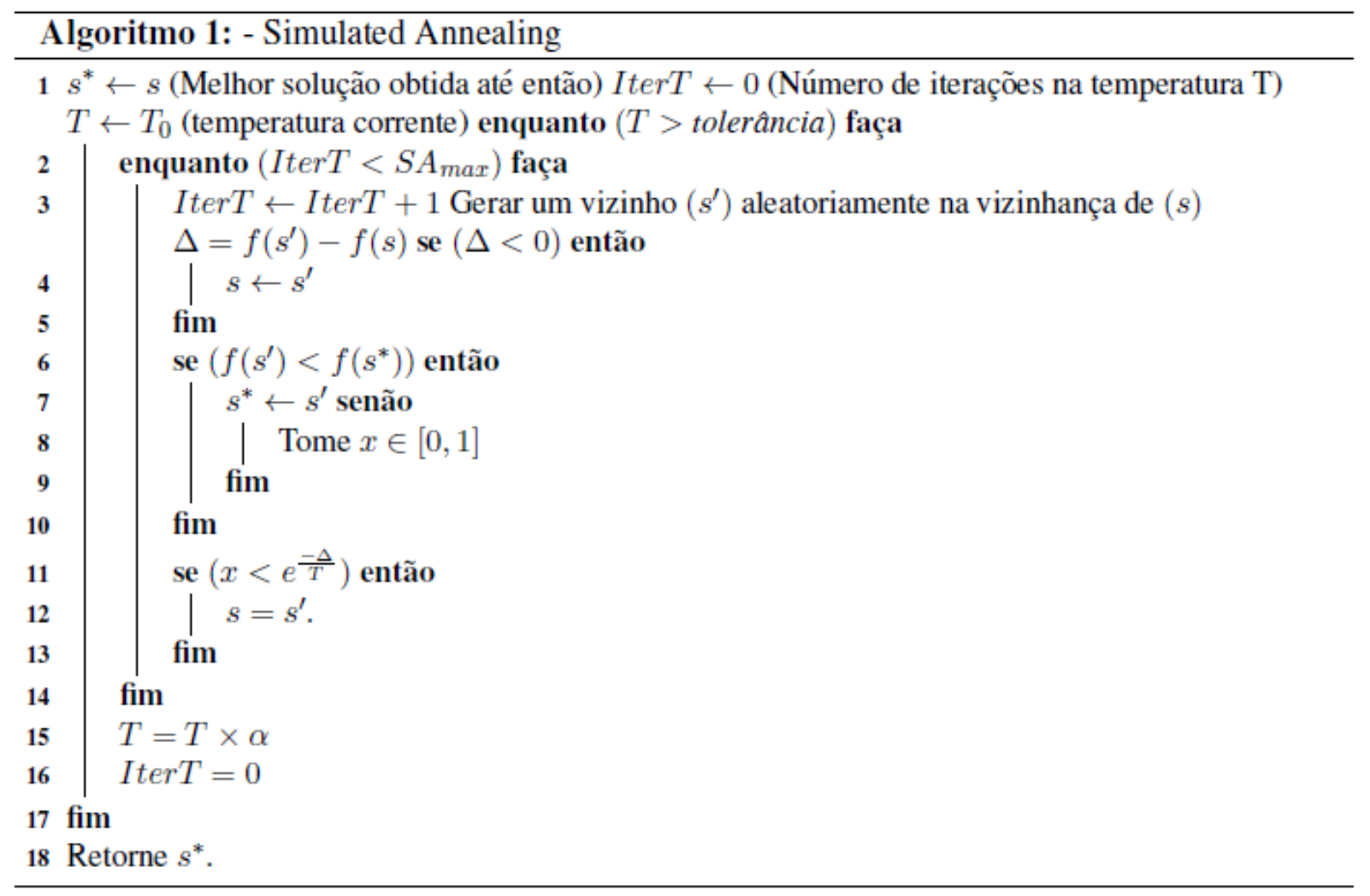

Figura 5. Algoritmo do Método Simulated Annealing.

\subsection{O Método Hooke Jeeves}

O Método Hooke Jeeves, proposto por HOOKE e JEEVES (1961) é um algoritmo determinístico de busca local.

Como é mostrado em OLIVEIRA e SOUZA (2016), o método de Hooke Jeeves promove duas buscas ao longo de suas iterações.

Nos passos 1 e 2 mostrados no algoritmo, é realizado a primeira busca, a busca exploratória. Por exemplo, para uma função de duas variáveis, o método parti de um ponto inicial, e explora todas as direções coordenadas de busca (norte, sul, leste e oeste) para cada uma das variáveis. Assim, a direção onde a função objetivo tem seu valor diminuído, é a direção utilizada pelo método. Logo, nos dois primeiros passos é definido um novo ponto onde ocorre a melhora no valor da função objetivo. Após a busca ao longo das direções coordenadas, o método executa a próxima etapa, descrita nos passos 3 e 4 . A busca padrão, que também é conhecida como de progressão ou aceleração, é uma busca que avança na melhor direção definida na execução da primeira busca exploratória, até um valor $\alpha>0$ (fator de aceleração). Após este avanço, o método prossegue com seus passos até que a tolerância desejada seja alcançada. Assim o método é finalizado. 
Em SILVA (2011), é possível encontrar um algoritmo para o método de Hooke Jeeves, onde são detalhados os passos do método e as buscas que o método realiza.

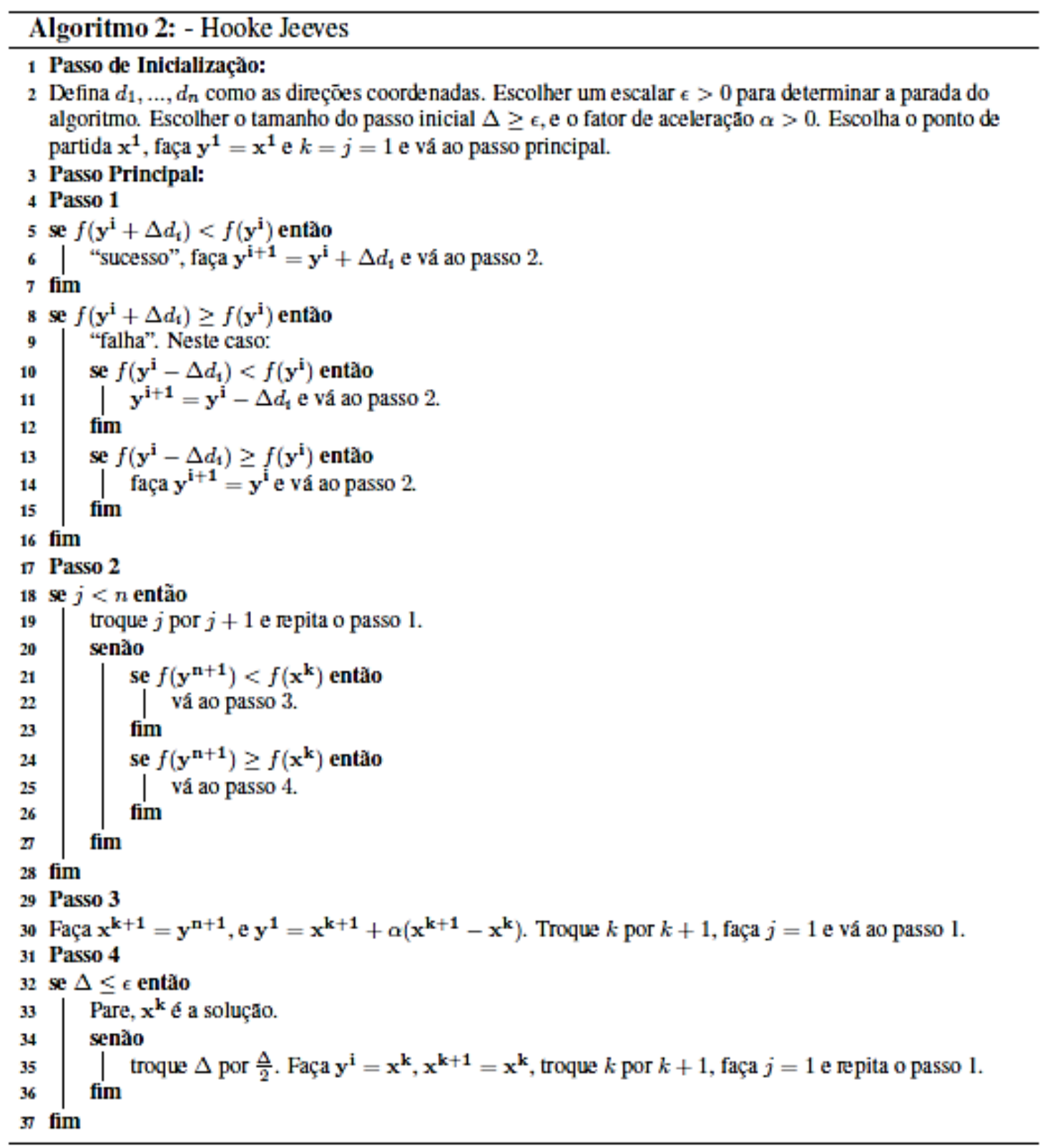

Figura 6. Algoritmo do Método Hooke Jeeves.

A seguir, a Figura 7 ilustra os passos do método.

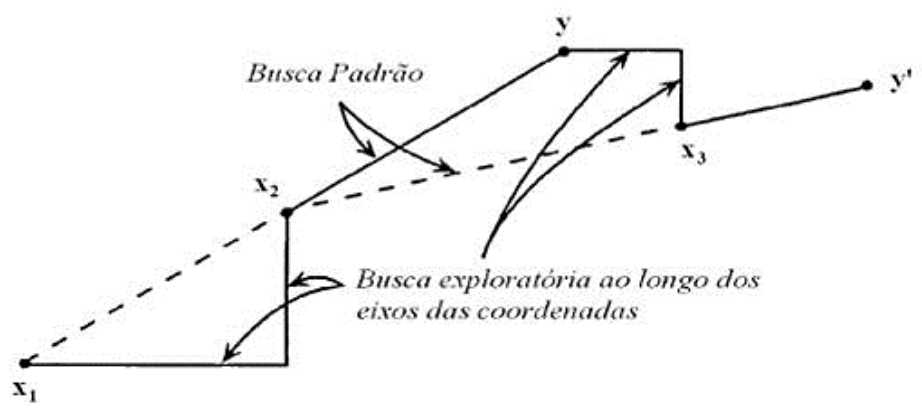

Figura 7. Deslocamentos do Método Hooke Jeeves. 


\subsection{O Método Híbrido - Simulated Annealing/Hooke Jeeves}

O método híbrido Simulated Annealing/Hooke Jeeves, visa inserir o método Hooke Jeeves $(\mathrm{HJ})$ ao longo das iterações do método Simulated Annealing (SA). Quando uma solução é gerada pelo método SA, seguindo os critérios de aleatoriedade do método, é feita uma busca com o método HJ nesta solução, após o processo de aceite deste novo ponto gerado. O método HJ realiza seus passos, buscando melhorar a solução gerada pelo método SA. Se uma melhora é realizada no ponto inicial gerado pelo método SA, esta nova solução é aceita e seguem-se os passos do método Simulated Annealing, realizando a redução de temperatura e reiniciando a iteração. Caso contrário, a solução gerada pelo SA é mantida, seguindo o procedimento normal.

Portanto, em cada ponto aceito pelo método SA é realizada uma busca local por meio do método HJ. Essa busca realizada visa melhorar a qualidade da solução gerada pelo Simulated Annealing nas proximidades da região onde este ponto (solução) gerado se encontra.

A seguir, tem-se o algoritmo do método híbrido SA/HJ.

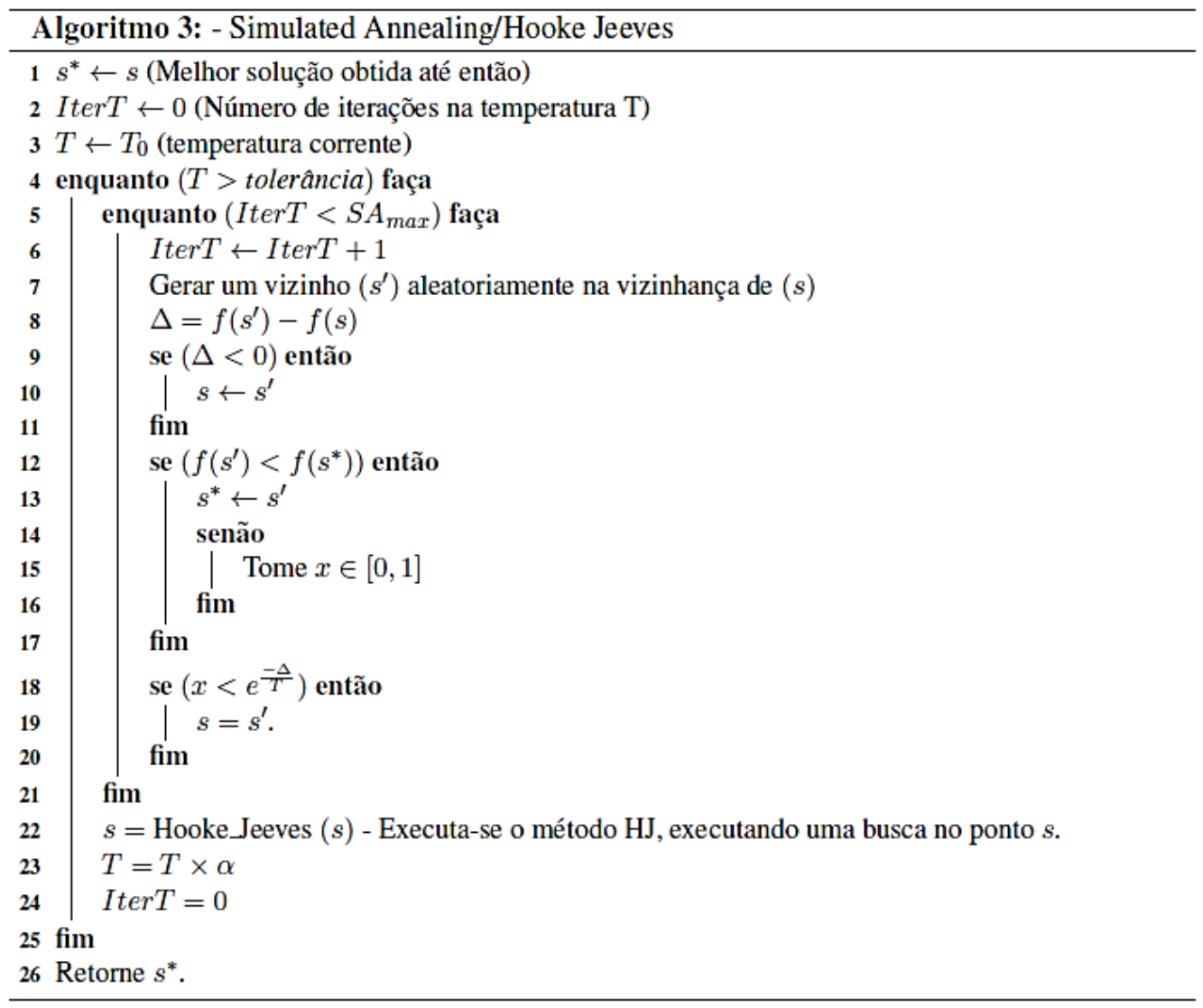

Figura 8. Algoritmo do Método Simulated Annealing/Hooke Jeeves. 


\section{RESULTADOS E DISCUSSÁO}

Apresentam-se agora os resultados obtidos na minimização das funções não lineares, por meio da metodologia proposta. Os resultados baseiam-se na demonstração do tempo computacional que os métodos Simulated Annealing e Simulated Annealing/Hooke Jeeves demoraram para obter o minimizador global de cada uma das funções não lineares aqui trabalhadas.

Por se tratar de um problema de minimização global, o método Simulated Annealing facilita com que ocorra o distanciamento de mínimos locais, com a devida aproximação a mínimos globais. Assim, o método Hooke Jeeves trabalha, buscando refinar a região onde está a solução gerada pelo método SA. Este refinamento pode influenciar no tempo computacional gasto na realização do problema proposto.

$\mathrm{Na}$ Tabela 1 pode-se ver estes resultados. Foram feitos testes para as quatro funções mostradas, considerando dois valores para a dimensão do problema.

Tabela 1. Tempo computacional para obtenção dos mínimos globais das funções.

\begin{tabular}{c|c|c}
\hline Metodologia & SA & SA/HJ \\
\hline Funções & \multicolumn{2}{|c}{ Tempo Computacional (s) } \\
\hline Ackley - 20 variáveis & 1,0940 & 1,0470 \\
Ackley - 30 variáveis & 2,1330 & 2,0470 \\
Levy - 20 variáveis & 1,8910 & 1,7660 \\
Levy - 30 variáveis & 3,8460 & 3,6090 \\
Schwefel - 20 variáveis & 0,9060 & 0,8750 \\
Schwefel - 30 variáveis & 1,7230 & 1,6090 \\
Styblinski-Tang - 20 variáveis & 1,2350 & 1,1570 \\
Styblinski-Tang - 30 variáveis & 2,5010 & 2,3440 \\
\hline
\end{tabular}

Percebe-se, na Tabela 1, que o tempo computacional gasto para a obtenção dos minimizadores de todas as funções não lineares trabalhadas foi inferior para o método híbrido. Os dois métodos foram eficientes pois obtiveram com facilidade os mínimos globais das funções. No entanto, o método híbrido demonstrou ser mais rápido para a resolução desse problema.

Além do tempo médio computacional, foram obtidos os também os resultados referentes ao número médio de iterações que cada um dos métodos trabalhados utilizou na obtenção dos minimizadores globais das funções testadas. Os resultados encontram-se na Tabela 2. 
Tabela 2. Número de iterações para obtenção dos mínimos globais das funções.

\begin{tabular}{c|c|c}
\hline Metodologia & SA & SA/HJ \\
\hline Funções & \multicolumn{2}{|c}{ Número de Iterações } \\
\hline Ackley - 20 variáveis & 99 & 75 \\
Ackley - 30 variáveis & 103 & 78 \\
Levy - 20 variáveis & 99 & 73 \\
Levy - 30 variáveis & 101 & 74 \\
Schwefel - 20 variáveis & 99 & 75 \\
Schwefel - 30 variáveis & 98 & 76 \\
Styblinski-Tang - 20 variáveis & 98 & 74 \\
Styblinski-Tang - 30 variáveis & 101 & 75 \\
\hline
\end{tabular}

Com os resultados presentes na Tabela 2 percebe-se novamente melhores resultados para o método híbrido Simulated Annealing/Hooke Jeeves. Assim como ocorreu em relação ao tempo computacional, o número de iterações do método Simulated Annealing foi superior ao número de iterações do método híbrido.

\section{CONSIDERAÇOES FINAIS}

Com os resultados aqui apresentados, podemos concluir que os dois métodos aqui apresentados são eficientes na resolução do problema proposto, a minimização de funções não lineares de 20 e 30 variáveis. É possível perceber também, que o método híbrido obteve o mínimo das funções em um tempo menor do que o método Simulated Annealing. Apesar de ser pequena esta diferença, podemos destacar que a inserção do método Hooke Jeeves ao longo das iterações do método SA possibilitou uma melhora nos resultados obtidos. Também é possível perceber a diferença em relação ao número de iterações entre os métodos. O número de iterações do método híbrido para obter os minimizadores foi inferior ao método principal Simulated Annealing. Este fato também destaca a eficiência da hibridização Simulated Annealing/ Hooke Jeeves.

Portanto, além de ser eficiente na minimização das funções não lineares, o método híbrido aqui proposto mostrou um ótimo desempenho para este problema trabalhado.

\section{REFERÉNCIAS}

BACK, Thomas. Evolutionary algorithms in theory and practice: evolution strategies, evolutionary programming, genetic algorithms. Oxford university press, 1996.

COSTA, M. F. Aplicação de Meta-heurísticas no Escalonamento de Motoristas para o 
Transporte de Madeira. 2012. Dissertação de Mestrado. Universidade Federal do Espírito Santo.

HOOKE, Robert; JEEVES, Terry A. Direct Search Solution of Numerical and Statistical Problems. Journal of the ACM (JACM), v. 8, n. 2, p. 212-229, 1961.

KIRKPATRICK, Scott; GELATT, C. Daniel; VECCHI, Mario P. Optimization by simulated annealing. science, v. 220, n. 4598, p. 671-680, 1983.

METROPOLIS, Nicholas et al. Equation of state calculations by fast computing machines. The journal of chemical physics, v. 21, n. 6, p. 1087-1092, 1953.

Molga, M., I\& Smutnicki, C. (2005), Test functions for optimization needs. Disponível em: <www.zsd.ict.pwr.wroc.pl/files/docs/functions.pdf.> Acesso em junho 2017.

Oliveira, M. B., I\& Souza, J. S. Determinação de minimizadores globais de funções por meio de métodos híbridos com base no método Luus Jaakola. In: Anais do XLVIII SBPO, Simpósio Brasileiro de Pesquisa Operacional, Vitória, 2016.

Silva, M. R. Um Novo Método Híbrido Aplicado à Solução de Sistemas Não-Lineares com Raízes Múltiplas" In: XLIII Simpósio Brasileiro de Pesquisa Operacional - SBPO, Ubatuba - SP, 2011. 